\title{
Retinal fractal dimension is increased in persons with diabetes but not impaired glucose metabolism: the Australian Diabetes, Obesity and Lifestyle (AusDiab) study
}

\author{
J. W. Y. Yau • R. Kawasaki • F. M. A. Islam • J. Shaw • \\ P. Zimmet • J. J. Wang • T. Y. Wong
}

Received: 18 February 2010 / Accepted: 30 April 2010/Published online: 5 June 2010

(C) Springer-Verlag 2010

\begin{abstract}
Aims/hypothesis The fractal dimension $\left(D_{\mathrm{f}}\right)$ of the retinal vasculature is a global measure of its branching pattern complexity. We examined the relationship of retinal $D_{\mathrm{f}}$ with diabetes.

Methods We conducted a cross-sectional study of 1,577 participants with diabetes and impaired glucose metabolism and normal controls from the population-based Australian Diabetes, Obesity and Lifestyle (AusDiab) study. Retinal $D_{\mathrm{f}}$ was quantified from fundus photographs using a computerbased programme and diabetes status was determined by
\end{abstract}

J. W. Y. Yau $\cdot$ R. Kawasaki $\cdot$ F. M. A. Islam · J. J. Wang •

T. Y. Wong $(\bowtie)$

Centre for Eye Research Australia, University of Melbourne,

32 Gisborne Street,

East Melbourne 3002 VIC, Australia

e-mail: twong@unimelb.edu.au

J. W. Y. Yau

Royal Victorian Eye and Ear Hospital,

Melbourne, VIC, Australia

F. M. A. Islam

Department of Mathematics and Computing,

The University of Southern Queensland,

Toowoomba, QLD, Australia

J. Shaw $\cdot$ P. Zimmet

Baker IDI Heart and Diabetes Institute,

Melbourne, VIC, Australia

J. J. Wang

Centre for Vision Research, University of Sydney,

Sydney, NSW, Australia

T. Y. Wong

Singapore Eye Research Institute, Singapore National Eye Centre,

Singapore, Republic of Singapore oral glucose tolerance test based on the WHO criteria.

Results After adjustment for age, sex and vascular risk factors, persons with higher retinal $D_{\mathrm{f}}$ were more likely to have diabetes (OR 1.56; 95\% CI 1.14-2.14, highest vs lowest fractal tertile). This relationship remained with further adjustment for retinal arteriolar calibre and presence of retinopathy (OR 1.64; 95\% CI 1.19-2.27), and after excluding participants with retinopathy (OR 1.60; 95\% CI 1.16-2.21). Retinal $D_{\mathrm{f}}$ was not related to impaired glucose tolerance or impaired fasting glucose (OR 1.19; 95\% CI 0.85-1.67).

Conclusions/interpretation Individuals with diabetes, but not with impaired glucose metabolism, have greater retinal $D_{\mathrm{f}}$, reflecting greater complexity of the retinal vasculature. Our findings suggest the presence of early microvascular changes in the retinal vasculature of persons with diabetes, even in the absence of overt retinopathy.

Keywords Diabetes - Diabetic retinopathy

Fundus photographs - Impaired glucose metabolism ·

Microvascular changes $\cdot$ Retinal fractal dimension

\author{
Abbreviations \\ AusDiab Australian Diabetes, Obesity and Lifestyle \\ $D_{\mathrm{f}} \quad$ Fractal dimension \\ DR Diabetic retinopathy \\ 2HPG $2 \mathrm{~h}$ plasma glucose
}

\section{Introduction}

Recent studies have shown that alterations in the retinal architecture, as reflected by variations in retinal vascular calibre, may be a marker of prediabetes, diabetes and its microvascular complications [1-3]. Retinal vessel calibre 
measurements, however, offer information on a single specific variable of the retinal vascular network.

Fractal dimensions $\left(D_{\mathrm{f}}\right)$ represent the complexity of geometric patterns such as the retinal vasculature with selfsimilar branching pattern. Fractal analysis has been applied to characterise self-similar structures in various organs and tissues, such as the bronchial tree and the vascular system of the myocardium and kidney. The $D_{\mathrm{f}}$ of the retinal vasculature can now be measured from digital fundus photographs using computer programs [4]. Recent studies show that changes in retinal $D_{\mathrm{f}}$ are associated with diabetic retinopathy $[5,6]$ and hypertension [4], with a suggestion from a small study of an association with diabetes [7]. Few studies have examined the possible association of retinal $D_{\mathrm{f}}$ in people with impaired glucose metabolism. In this study, we compared the $D_{\mathrm{f}}$ of people with diabetes and impaired glucose metabolism with normal, non-diabetic individuals.

\section{Methods}

Study population Study participants were recruited from the Australian Diabetes, Obesity and Lifestyle (AusDiab) study. Detailed methodology of this study has been described elsewhere [8]. In brief, the AusDiab study surveyed adults aged $\geq 25$ years from 42 randomly selected areas in Australia. At the baseline examination in 19992000, all participants $(n=11,247)$ underwent an OGTT and diabetes was diagnosed based on the 1999 WHO classification. Participants with a fasting plasma glucose (FPG) $\geq 7.0 \mathrm{mmol} / \mathrm{l}$, or a $2 \mathrm{~h}$ plasma glucose (2HPG) level $\geq 11.1 \mathrm{mmol} / \mathrm{l}$, were classified as having diabetes. Impaired glucose tolerance (IGT) was defined as FPG $<7.0 \mathrm{mmol} / \mathrm{l}$ and $2 \mathrm{HPG}$ of $\geq 7.8$ and $<11.1 \mathrm{mmol} / \mathrm{l}$, and impaired fasting glucose (IFG) was defined as FPG of $\geq 6.1$ and $<7.0 \mathrm{mmol} / 1$ and $2 \mathrm{HPG}$ of $<7.8 \mathrm{mmol} / \mathrm{l}$.

Of the original cohort $(n=11,247)$, all participants with diabetes, IFG or IGT, and a random sample of those with normal glucose metabolism, were invited to participate in a substudy of diabetic complications. Of the 2,762 participants in this substudy, 1,848 (67\%) attended the follow-up study in 2004-2005. We used digital retinal photographs taken at the 5 year follow-up visit of the study. Of the 1,848 participants, we excluded $77(4 \%)$ participants without gradable vessel calibre and 194 (10\%) without gradable photographs for $D_{\mathrm{f}}$, leaving 1,577 participants for this analysis. Compared with the participants with gradable photographs, those with ungradable photographs were older (67 vs 61 years), more likely to have diabetes $(46 \%$ vs $32 \%)$ and retinopathy $(9.4 \%$ vs $4.2 \%)$, but less likely to have IFG $(6.7 \%$ vs $21.3 \%)$. There was no sex difference between the two groups. Institu- tional board review approval was obtained and all participants gave informed consent.

Retinal photography and fractal analysis At the 5 year follow-up visit, two-field retinal photographs (macular and disc-centred) were taken of both eyes using a non-mydriatic digital retinal camera. Two trained graders, masked to patients' clinical diagnoses, graded the disc-centred fundus photographs using a validated semi-automated computer program described previously $[4,6]$. In brief, the program automatically traces all retinal vessels within a predefined circular region, 3.5 optic disc radii, centred on the optic disc. The grader then checks the trace against the original photograph and removes the occasional artefact misidentified as a vessel (e.g. peripapillary atrophy, pigment abnormalities, choroidal vessels and nerve fibre reflection). The program then calculates the $D_{\mathrm{f}}$ from the refined line tracing using the box-counting approach. Grading time for each photograph is approximately $5 \mathrm{~min}$ [4]. Reproducibility of measurements was very high, with intra- and intergrader intraclass correlation coefficients ranging from 0.93 to 0.95 [4]. We additionally graded for retinopathy signs according to the simplified Wisconsin grading system [9].

Statistical analysis We used multinomial logistic regression models to determine the likelihood (OR) of having diabetes or IFG/IGT in relation to $D_{\mathrm{f}}$, and defined the lowest tertile as the reference group versus the remaining two upper tertiles. We constructed four models: Model 1 included adjustments for age and sex; Model 2 included additional adjustments for systolic blood pressure, BMI and cholesterol; Model 3 included additional adjustments for retinal arteriolar calibre and retinopathy, which have been associated with higher $D_{\mathrm{f}}$ in previous studies [6]; and in Model 4 we adjusted for variables in Model 2 after excluding people with retinopathy signs.

\section{Results}

Of the 1,577 participants, 797 (50.5\%) were female, and the mean age (standard deviation) of the participants was 61 (12) years. Participants with normal glucose tolerance were younger (58 years) than those with IGT/IFG (62 years) and diabetes (64 years). Of the 498 participants with diabetes, only 13 had type 1 diabetes.

Table 1 shows that, after adjustment for age and sex, increased $D_{\mathrm{f}}$ (highest vs lowest tertile) was associated with diabetes (OR 1.50; 95\% CI 1.12-2.02). The association remained similar after further adjustment for vascular risk factors (OR 1.56; 95\% CI 1.14-2.14). There was no significant association of retinal $D_{\mathrm{f}}$ with IFG/IGT (OR $1.19 ; 95 \%$ CI $0.85-1.67)$. In supplementary analyses, the 
Table 1 Association of fractal dimension with impaired glucose metabolism and diabetes

\begin{tabular}{|c|c|c|c|c|}
\hline Model & Retinal fractal dimension & Normal $(n=743,46 \%)$ & IGT/IFG $(n=336,22 \%)$ & Diabetes $(n=498,32 \%)$ \\
\hline \multirow[t]{2}{*}{ Model $1^{\mathrm{a}}$} & Tertile 2: $1.435-1.455$ & 1.00 & $0.79(0.55-1.11)$ & $1.02(0.76-1.40)$ \\
\hline & Tertile $3:>1.455$ & 1.00 & $1.19(0.86-1.64)$ & $1.50(1.12-2.02)$ \\
\hline \multirow[t]{2}{*}{ Model $2^{b}$} & Tertile 2: $1.435-1.455$ & 1.00 & $0.77(0.54-1.10)$ & $0.97(0.69-1.35)$ \\
\hline & Tertile $3:>1.455$ & 1.00 & $1.19(0.85-1.67)$ & $1.56(1.14-2.14)$ \\
\hline \multirow[t]{2}{*}{ Model $3^{\mathrm{c}}$} & Tertile 2: $1.435-1.455$ & 1.00 & $0.81(0.56-1.18)$ & $1.02(0.71-1.44)$ \\
\hline & Tertile $3:>1.455$ & 1.00 & $1.23(0.87-1.74)$ & $1.64(1.19-2.27)$ \\
\hline \multirow[t]{2}{*}{ Model $4^{\mathrm{d}}$} & Tertile 2: $1.435-1.455$ & 1.00 & $0.77(0.53-1.11)$ & $0.99(0.70-1.40)$ \\
\hline & Tertile $3:>1.455$ & 1.00 & $1.21(0.86-1.70)$ & $1.60(1.16-2.21)$ \\
\hline
\end{tabular}

Tertile $1(<1.435)$ is the reference group

${ }^{a}$ Model 1: odds ratio $(95 \% \mathrm{CI})$ adjusted for age and sex

${ }^{\mathrm{b}}$ Model 2: odds ratio $(95 \% \mathrm{CI})$ adjusted for age, sex, systolic blood pressure, BMI and cholesterol

${ }^{\mathrm{c}}$ Model 3: odds ratio $(95 \% \mathrm{CI})$ adjusted for factors in Model 2 plus retinopathy and retinal vascular calibre

${ }^{\mathrm{d}}$ Model 4: odds ratio $(95 \% \mathrm{CI})$ adjusted for factors in Model 2 after excluding participants with retinopathy IFG, impaired fasting glucose; IGT, impaired glucose tolerance

association between retinal $D_{\mathrm{f}}$ and diabetes remained similar after additional adjustment for retinal arteriolar calibre and presence of retinopathy (OR 1.64; 95\% CI $1.19-2.27$ ) and in people without retinopathy (OR 1.60; 95\% CI 1.16-2.21).

There was no significant association between $D_{\mathrm{f}}$ and diabetic retinopathy (DR) severity, with $D_{\mathrm{f}}$ in eyes with minimum DR 1.442, moderate to severe DR 1.441, and proliferative DR 1.403 ( $p$ value for trend=0.19).

\section{Discussion}

Our study shows that persons with diabetes had increased retinal $D_{\mathrm{f}}$, independent of age, sex, vascular risk factors, retinal vascular calibre and diabetic retinopathy. In contrast, there was no significant increase in people with impaired glucose metabolism. These findings suggest that increased complexity of the retinal microvascular structure may be a sign of early diabetes-related alteration in the microcirculation.

Our findings support data from only one previous study that reported a positive association between persons with diabetes and increased retinal $D_{\mathrm{f}}$ [7]. The objective of this previous study, however, was to evaluate the technical and methodological aspects of fractal analysis as applied to persons with diabetes; hence it was limited by a small sample size and the absence of an OGTT to determine glycaemic status. Apart from a consistent cross-sectional association between diabetic retinopathy and increased retinal $D_{\mathrm{f}}[5,6]$, there are few data on the relationship with diabetes, and none on IGT/IFG. A subsequent report [10] from the same study sample [6] showed no longitudinal association of retinal $D_{\mathrm{f}}$ with incident diabetic retinopathy, suggesting that the observed increase in $D_{\mathrm{f}}$ in patients with diabetic retinopathy is a consequent change.

The pathophysiological mechanisms underlying our findings remain unclear. It has been shown that, in pathological states, the retinal vasculature remodels in response to release of angiogenic factors to ensure that all tissues are adequately perfused. Alterations in retinal vessel patterns may be a response to hypoxia, and are typically seen in advanced stages as retinal neovascularisation, as in proliferative diabetic retinopathy. However, our observation of an association between retinal vascular fractals and diabetes in the absence of even mild retinopathy suggests that increased $D_{\mathrm{f}}$ in persons with diabetes is not due to proliferative diabetic disease, but that alterations in retinal vascular geometric complexity are an indicator of much earlier subclinical vascular pathology.

It has been further suggested in experimental studies that diabetes induces a microvascular remodelling process that alters the structural morphology of retinal capillaries [11]. It is possible that increased retinal $D_{\mathrm{f}}$ may be a reflection of these subtle morphological alterations, detectable before characteristic vasculopathy manifests. Finally, according to the Murray principle, the retinal architecture is structurally developed to optimise flow, and deviations from this optimal state occur in disease processes. Based on this hypothesis, increased $D_{\mathrm{f}}$ may reflect alterations in the retinal architecture caused by hyperglycaemia, which may render the retinal vessels more vulnerable to further damage by other vascular risk factors.

The strengths of our study include its large sample size, the recruitment of participants from a population-based cohort with comprehensive data on risk factors and retinopathy status, and the standardised and masked assessment of retinal $D_{\mathrm{f}}$. Potential limitations include its 
cross-sectional design, which limits our ability to draw inferences on temporality of observed associations. The fact that there was no significant association with IGT/IFG suggests that such changes are more likely to be consequent than antecedent to diabetes.

In summary, we show that people with diabetes are more likely to have increased complexity of their retinal microvasculature, as quantified by higher retinal $D_{\mathrm{f}}$ even in the absence of overt retinopathy. In contrast, retinal $D_{\mathrm{f}}$ was not related to IGT/IFG. Further prospective studies are needed to confirm these findings, to improve our understanding of the pathophysiological pathways of early vascular structural alterations in diabetes, and to determine the potential clinical utility of fractal analysis as a novel means to detect early subclinical diabetic microvasculopathy.

Conflict of interest The authors declare that there is no duality of interest associated with this manuscript.

\section{References}

1. Grauslund J, Hodgson L, Kawasaki R, Green A, Sjolie AK, Wong TY (2009) Retinal vessel calibre and micro- and macrovascular complications in type 1 diabetes. Diabetologia 52: 2213-2217
2. Tikellis G, Wang JJ, Tapp R et al (2007) The relationship of retinal vascular calibre to diabetes and retinopathy: the Australian Diabetes, Obesity and Lifestyle (AusDiab) study. Diabetologia 50:2263-2271

3. Nguyen TT (2007) Retinal vascular changes in pre-diabetes and prehypertension - new findings and their research and clinical implications. Diabetes Care 30:2708-2715

4. Liew G, Wang JJ, Cheung $\mathrm{N}$ et al (2008) The retinal vasculature as a fractal: methodology, reliability, and relationship to blood pressure. Ophthalmology 115:1951-1956

5. Daxer A (1993) The fractal geometry of proliferative diabetic retinopathy: implications for the diagnosis and the process of retinal vasculogenesis. Curr Eye Res 12:1103-1109

6. Cheung N, Donaghue KC, Liew G et al (2009) Quantitative assessment of early diabetic retinopathy using fractal analysis. Diabetes Care 32:106-110

7. Cheng SC, Huang YM (2003) A novel approach to diagnose diabetes based on the fractal characteristics of retinal images. IEEE Trans Inf Technol Biomed 7:163-170

8. Dunstan DW, Zimmet PZ, Welborn TA et al (2002) The Australian Diabetes, Obesity and Lifestyle Study (AusDiab) methods and response rates. Diabetes Res Clin Pract 57:119-129

9. Aldington SJ, Kohner EM, Meuer S, Klein R, Sjolie AK (1995) Methodology for retinal photography and assessment of diabetic retinopathy: the EURODIAB IDDM complications study. Diabetologia 38:437-444

10. Lim SW, Cheung N, Wang JJ et al (2009) Retinal vascular fractal dimension and risk of early diabetic retinopathy: a prospective study of children and adolescents with type 1 diabetes. Diabetes Care 32:2081-2083

11. Dosso AA, Leuenberger PM, Rungger-Brandle E (1999) Remodeling of retinal capillaries in the diabetic hypertensive rat. Invest Ophthalmol Vis Sci 40:2405-2410 